\section{Surveillance of intraocular foreign bodies in the UK}

\begin{abstract}
Aims To estimate the incidence of penetrating injuries with retained intraocular foreign bodies (IOFBs) in the United Kingdom, and to provide epidemiological data on the aetiology, management, and visual outcome of such injuries.

Methods Cases were identified prospectively by active surveillance through the British Ophthalmological Surveillance Unit reporting card system, for the 12-month period June 2004 to May 2005 inclusive. Questionnaire data were obtained from UK ophthalmologists at presentation and 6 months following presentation.
\end{abstract}

Results Data were available on 97 patients at presentation and 95 patients at follow-up. The minimum estimated incidence of IOFBs in the United Kingdom identified in this study was 0.16 per 100000 . All patients were male. Hammering was the most common mechanism of injury, occurring in $62 \%$ of patients. The IOFB was found in the anterior segment in $24 \%$, the posterior segment in $73 \%$, and involved both segments in $3 \%$.

Endophthalmitis was diagnosed in $9 \%$ of patients. Best-corrected visual acuity of the injured eye at final follow-up was $6 / 12$ or better in $67 \%, 6 / 18$ to $6 / 60$ in $11 \%$, and worse than $6 / 60$ in $22 \%$. Prognostic factors for a poor visual outcome included poor visual acuity at presentation, prolapse of intraocular tissue, development of endophthalmitis, development of retinal detachment, and large size of IOFB.

Conclusions The incidence of IOFB in the United Kingdom appears to have reduced compared to previous studies. The majority of patients $(67 \%)$ retain good visual acuity in the injured eye; however, a significant minority (22\%) sustain long-term severe visual loss. Eye (2008) 22, 1141-1147; doi:10.1038/sj.eye.6702868; published online 25 May 2007
FR Imrie ${ }^{1}$, A Cox ${ }^{1}$, B Foot ${ }^{2}$ and CJ MacEwen ${ }^{1}$

Keywords: intraocular foreign body;

surveillance; penetrating injury;

endophthalmitis; prognostic factors

\section{Introduction}

In the 1980s and 1990s, intraocular foreign bodies (IOFBs) were identified as a significant cause of serious ocular trauma, accounting for $15-25 \%$ of penetrating eye injuries. ${ }^{1-4}$ IOFBs frequently cause severe visual loss, with 17-42\% resulting in final vision worse than $6 / 60$ in the injured eye..$^{3-8}$ A high percentage $(50-54 \%)$ of IOFBs were identified as arising from work-related injuries, $3,6,9$ and regardless of the environment in which the injury occurred,

hammering was the most common mechanism of injury, accounting for $71-80 \%$ of such injuries. ${ }^{7,10-12}$ In one large American study, only $6 \%$ of persons with IOFBs were wearing protective eyewear. ${ }^{9}$ The UK Health and Safety at Work Act (1974) places a duty on employers to provide appropriate protective eyewear, and a duty on employees to wear such protective eyewear where provided. Through estimating the incidence and aetiology of IOFBs in the United Kingdom, we hoped to determine whether this largely preventable form of eye injury remained a significant problem, and whether further measures to reduce its incidence could be suggested.

To date, the majority (59-88\%) of IOFBs caused damage to or were located in the posterior segment. ${ }^{3,6-8,11,12}$ There is debate in the literature regarding the best management of posterior segment IOFBs. Some authors favour an initial attempt of magnetic extraction of the material, ${ }^{11}$ whereas others prefer posterior vitrectomy with removal of the IOFB using forceps. ${ }^{10}$ This study aimed to determine the prevailing practice of UK ophthalmologists.
'Department of Ophthalmology, Ninewells Hospital and Medical School, Dundee, UK

${ }^{2}$ The Royal College of Ophthalmologists, London, UK

Correspondence: FR Imrie, Department of

Ophthalmology, Gartnavel General Hospital, Great Western Road, Glasgow G12 OYN, UK Tel: + 44141211 1040; Fax: +44 1412112054 .

E-mail: fraser.imrie@

btopenworld.com

Received: 30 January 2007 Accepted in revised form: 26 April 2007 Published online: 25 May 2007

Proprietary interests: none

This work was presented at the Royal College of Ophthalmologists Annual Congress, May 2006 
Several factors have been shown to be associated with a poor visual outcome following penetrating injury with IOFB. These include large diameter of IOFB, poor visual acuity on presentation, relative afferent pupil defect (RAPD) on presentation, corneoscleral entry wound, uveal prolapse, vitreous haemorrhage, and presence of a secondary retinal detachment. ${ }^{6-8,10,13,14}$ The study aimed to determine whether the final visual outcome had improved compared to previous reports and whether previously identified prognostic factors remained predictive of a poor outcome.

To date, there has been no previous UK-wide prospective survey of IOFBs. This study was undertaken to assess the current incidence, aetiology, management, and outcome of IOFBs in the UK.

\section{Materials and methods}

This was a population-based, prospective study with active surveillance of cases through the British Ophthalmological Surveillance Unit (BOSU) monthly reporting card system. ${ }^{15}$ The BOSU was established to facilitate research into rare eye conditions with significant public health or scientific importance. Individual investigators submit proposals to BOSU for conditions to be surveyed and the BOSU steering committee determines which conditions will be surveyed - usually three or four at any particular time. All consultant or associate specialist ophthalmologists with clinical autonomy in the United Kingdom form the reporting base for the BOSU surveillance scheme. Each month they are sent a reporting card by BOSU requesting them to report if they have treated in the preceding month any patient with the conditions currently under surveillance. The BOSU then informs the respective study investigators which ophthalmologists have reported a case and the study investigators then contact the reporting ophthalmologist.

The Multi-Centre Research Ethics Committee for Scotland (Committee A) reviewed this study and gave approval in April 2004.

For the 12-month study period between June 2004 and May 2005 inclusive, ophthalmologists were asked to notify the study investigators, through BOSU, of any case of IOFB newly presenting to them. IOFB was defined as a penetrating (single, full-thickness) eye injury with retained foreign material anywhere within the globe. Perforating (through and through) injuries and orbital foreign bodies were excluded.

Following case notification to the BOSU, every reporting ophthalmologist was sent a detailed questionnaire by the study investigators requesting data on the aetiology, presenting features, and initial management of the IOFB. Six months following presentation, reporting ophthalmologists were sent a follow-up questionnaire requesting data on subsequent progress and outcome. Ophthalmologists who did not return a questionnaire were sent a reminder letter 2 and 3 months after the initial questionnaire was sent.

Data were entered onto an SPSS for Windows spreadsheet and data analysis was performed using this program. Visual acuity data were recorded in Snellen format but for the purpose of linear regression analysis these were subsequently converted to logMar format. 'Counting Fingers' vision was recorded as logMar 2.0, 'Hand Motions' as logMar 2.48, 'Perception of Light' as 3.0, and 'Non-Perception of Light' as 4.0. Multivariate linear regression was performed to determine which factors were predictive of the final best-corrected visual acuity. A forced entry method was used and the following factors were included in the model: visual acuity at presentation, prolapse of intraocular tissue at presentation, location of entry wound, presence of an RAPD at presentation, endophthalmitis at any time, location of IOFB, removal of IOFB within $24 \mathrm{~h}$, retinal detachment at any time, diameter of IOFB, and use of an external magnet to remove the IOFB.

\section{Results}

\section{$B O S U$ reports and estimates of $U K$ incidence of IOFB}

From June 2004 to May 2005 inclusive, 155 reports of patients with IOFB were made to BOSU by 104 ophthalmologists in the United Kingdom. Questionnaires were received from 134/155 reports (86.5\% response rate), of which 37 were excluded (13 were incorrectly diagnosed, 9 presented out with the study period, 8 were duplicate reports, and for 7 cases no details could be recalled by the reporting ophthalmologist). Questionnaire data were available on 97 patients at presentation and 95 patients at follow-up. Some variables analysed have $n<97$, as several questionnaires had incomplete data (particularly those cases referred to another unit for further treatment). As the main aim of the study was to estimate the incidence of IOFB in the United Kingdom, all reports sufficiently completed to meet the inclusion criteria were included in the analysis.

The current UK population is approximately 60 million. The reported incidence of IOFBs in the United Kingdom is therefore 97 per 60 million, which is equivalent to 0.16 per 100000 (95\% confidence interval $0.13-0.19$ per 100000 ).

\section{Patient characteristics and presenting details}

All the patients were male (97/97). The mean age at time of injury was 39 years (range 13-76 years) and $94 \%$ 
Table 1 Best-corrected visual acuity at initial presentation $(n=93)$ and final follow-up $(n=88)$

\begin{tabular}{lcc}
\hline Visual acuity & $\begin{array}{c}\text { Initial frequency } \\
(\mathrm{n}(\%))\end{array}$ & $\begin{array}{c}\text { Final frequency } \\
(\mathrm{n}(\%))\end{array}$ \\
\hline $6 / 12$ or better & $34(37)$ & $59(67)$ \\
$6 / 18$ to $6 / 60$ & $17(18)$ & $10(11)$ \\
Worse than $6 / 60$ & $42(45)$ & $19(22)$ \\
Total & $93(100)$ & $88(100)$ \\
\hline
\end{tabular}

(91/97) of patients were in the working age range (16-65 years). All injuries were unilateral, with 59\% (57/97) involving the right eye. The activity at the time of injury was paid employment in 62\% (60/97), do-it-yourself (DIY) in 13\% (13/97), leisure in 5\% (5/97), gardening in $2 \%(2 / 97)$, student work at university in $1 \%(1 / 97)$, assault in 1\% (1/97), and unknown in 13\% (13/97). The mechanism of injury was hammering in $62 \%(60 / 97)$, machine tools in $19 \%$ (18/97), firearms in $4 \%$ (4/97), other mechanisms in 10\% (10/97), and unknown in $4 \%$ (4/97). Protective eyewear was reportedly worn by $5 \%$ (5/97) and prescription spectacles by $2 \%$ (2/97).

Presentation to hospital occurred within $24 \mathrm{~h}$ of injury in 81\% (75/93), between 1 and 7 days in 10\% (9/93), between 7 days and 1 month in 4\% (4/93), between 1 and 6 months in 2\% (2/93) and after 6 months in 3\% (3/93). At presentation, $99 \%$ (94/95) of patients were phakic with only $1 \%(1 / 95)$ aphakic.

Visual acuity at presentation is presented in Table 1. In $54 \%$ (52/97), the IOFB was directly visible on clinical examination. Ocular imaging was performed in $83 \%$ (80/97) of cases: plain radiographs in 66\% (64/97), CT scan in 36\% (35/97), and ocular ultrasound in $21 \%$ (20/97). The site of entry of the IOFB was corneal in 65\% $(62 / 96)$, corneoscleral in 10\% (10/96), and scleral in $25 \%$ (24/96). A single IOFB was present in 93\% (88/95), and two or more were present in 7\% (7/95) of patients. The mean and median size of IOFB was 4.7 and $3.0 \mathrm{~mm}$, respectively (range $0.1-20.0$ ). The vast majority of IOFB were composed of metal $(91 \%(82 / 90))$. Only $8 / 90(9 \%)$ were non-metallic - glass $(2 / 90)$, stone $(1 / 90)$, concrete $(1 / 90)$, eyelashes $(1 / 90)$, and undetermined $(3 / 90)$. The IOFB was found in the anterior segment in $24 \%(22 / 90)$, the posterior segment in 73\% (65/90), and involved both segments in $3 \%(3 / 90)$. Table 2 presents the detailed position of the IOFB.

\section{Surgical details}

In two cases, no attempt was made to remove the IOFB as it was judged that the risks of surgery outweighed those of observation. In one case, a small splinter of masonry nail was embedded in the periphery of the lens, which
Table 2 Position of IOFB within the globe $(n=90)$

\begin{tabular}{lc}
\hline Position & $\begin{array}{c}\text { Frequency } \\
(\mathrm{n}(\%))\end{array}$ \\
\hline $\begin{array}{l}\text { Anterior segment }(n=22) \\
\text { Iris }\end{array}$ & $9(10)$ \\
Lens & $8(9)$ \\
Cornea and anterior chamber & $2(2)$ \\
Iridocorneal angle & $2(2)$ \\
Iris and lens & $1(1)$ \\
& \\
Anterior and posterior segment $(n=3)$ & \\
Cornea to retina & $1(1)$ \\
Anterior chamber and vitreous & $1(1)$ \\
Lens and vitreous & $1(1)$ \\
& \\
Posterior segment $(n=65)$ & \\
Surface of retina & $23(26)$ \\
Vitreous & $18(20)$ \\
Intraretinal & $14(16)$ \\
Ciliary body & $10(11)$ \\
Total & $90(100)$
\end{tabular}

remained clear. The other case involved an eyelash embedded in the lens, presenting as iritis 2 years following the initial injury.

The IOFB was removed within $24 \mathrm{~h}$ of injury in $43 \%$ $(35 / 82)$ of cases. The procedure to remove the IOFB was performed by vitreoretinal surgeons in $74 \%(64 / 87)$. The IOFB was removed by an anterior segment procedure in $29 \%(24 / 84)$ and by a procedure involving posterior segment surgery in $71 \%(60 / 84)$. Pars plana vitrectomy was the most frequently used technique for posterior segment IOFBs $(88 \%(53 / 60))$. In six cases, a posterior segment IOFB was removed via the anterior segment following pars plana vitrectomy. The external magnet was utilised in 17\% (10/60) of posterior segment cases: five cases in combination with pars plana vitrectomy and five cases in combination with sclerotomy. In two cases, a posterior segment IOFB was removed by sclerotomy and forceps alone.

\section{Endophthalmitis}

Endophthalmitis was suspected before IOFB removal in $15 \%$ (14/92) of patients. Intravitreal antibiotics were administered to $24 \%$ (22/92) of patients: 8 patients before IOFB removal and 14 patients at the time of IOFB removal. Endophthalmitis was subsequently diagnosed in $8 / 92(9 \%)$ patients, all of whom were suspected of having endophthalmitis before IOFB removal. Of these eight patients, seven received intravitreal antibiotics (two before IOFB removal and five at the procedure when the IOFB was removed). Positive cultures were obtained in only two cases (one case each of Haemophilus 
Table 3 Multivariate regression analysis using final best-corrected visual acuity as the dependent variable

\begin{tabular}{llccc}
\hline Variable & $\beta$ & 95\% confidence interval & t-Statistic & Significance \\
\hline LogMAR vision at presentation & 0.26 & $0.08-0.45$ & 2.85 & $>0.01$ \\
Endophthalmitis at any time & 1.3 & $0.06-1.10$ & 2.95 & $>0.01$ \\
Retinal detachment at any time & 0.77 & $0.30-1.25$ & 3.24 & $>0.01$ \\
Prolapse of intraocular tissue & 0.58 & $0.07-1.10$ & 2.24 & $>0.05$ \\
Diameter of IOFB & 0.14 & $0.01-0.11$ & 0.97 & $>0.05$ \\
Entry site involving sclera & 0.34 & $-0.06-0.75$ & -0.22 & 0.1 \\
Removal of IOFB within 24h & -0.05 & $-0.46-0.37$ & -0.46 & 0.83 \\
IOFB involves posterior segment & -0.1 & $-0.54-0.34$ & -0.3 & 0.65 \\
RAPD at time of injury & -0.11 & $-0.83-0.61$ & -0.65 & 0.76 \\
Removal with external magnet & -0.18 & $-0.72-0.36$ & & 0.52 \\
\hline
\end{tabular}

influenza and Staphylococcus epidermidis). Statistical analysis of factors predictive of endophthalmitis was not possible owing to the small number of cases.

\section{Outcome}

The mean time from injury to follow-up was 5 months (range 1-11 months). Table 1 presents the final visual acuity. The majority of patients $(67 \%$ (59/88)) maintained good best-corrected vision (6/12 or better) in the injured eye; however, 22\% (19/88) sustained severe visual loss (6/60 or worse). If best-corrected visual acuity is achieved with a pinhole or a lens that the patient does not wear, then day-to-day vision is poorer than bestcorrected vision. Using day-to-day vision to assess outcome, $34 \%(29 / 86)$ of patients had vision worse than $6 / 60$ in the injured eye.

At follow-up, phthsis had developed in 5\% (4/85), siderosis in $2 \%(2 / 85)$, and one eye had been enucleated (1\%). The final lens status was phakic in 36/85 (42\%), pseudophakic in 29/85 (34\%), and aphakic in 20/85 (24\%), meaning that the crystalline lens had been removed in 56\% (48/85) of patients at follow-up. Retinal detachment occurred in 33\% (29/88) of eyes, and in $16 \%$ $(14 / 86)$ the retina remained detached at follow-up. Silicone oil was used in $22 \%(19 / 86)$ of eyes and remained in situ in 17\% (15/86) at follow-up.

Table 3 presents the results of multivariate linear regression analysis using the final best-corrected visual acuity as the dependent variable. Factors associated with a poor visual outcome were poor visual acuity at presentation $(P \geqslant 0.01)$, development of endophthalmitis $(P \geqslant 0.01)$, development of retinal detachment $(P \geqslant 0.01)$, prolapse of intraocular tissue $(P \geqslant 0.05)$, and large size of IOFB $(P \geqslant 0.05)$.

\section{Discussion}

The incidence rate reported represents a minimum. This study has ascertained cases through the use of a well-established surveillance methodology shown to be more effective than other methods. ${ }^{16,17}$ However, it is likely that there is some degree of underascertainment. Previous reports for studies ascertaining cases through the BOSU have indicated that ascertainment rates usually lie between 75 and $95 \% .{ }^{15}$ Although not directly linked to ascertainment, response rates are the most common method for assessing underascertainment. ${ }^{18}$ The proportion of non-duplicate cases of IOFB that met the inclusion criteria is $97 / 134(72 \%)$. If all 155 reporting ophthalmologists had returned questionnaires, then 112 non-duplicate cases of IOFB would be expected $(72 \%$ of $155=112)$. The mean BOSU card return rate during the study period was $79 \%$. If all BOSU cards were returned, then 142 non-duplicate cases of IOFB would be expected $(112 / 0.79=142)$. Therefore, the corrected annual incidence of IOFB in the United Kingdom provided by this national, prospective study is 0.24 per 100000 . This is lower than estimates from previous UK regional studies (0.81 per 100000 in Northern Ireland ${ }^{7}$ from 1978 to 1986 and 0.59 per 100000 in Scotland ${ }^{2}$ from 1991 to 1992) and from a national study in Singapore from 1991 to 1996 (0.55 per $100000) .{ }^{1}$ The reduced incidence of IOFB in this study could be due to a reduction in penetrating injuries causing IOFB, under-reporting of cases, or a combination of both.

The BOSU reporting scheme is dependent on voluntary reporting of cases by participating ophthalmologists. Underascertainment of cases could be due to random errors (eg forgetting to report a case), reluctance to participate in the BOSU reporting scheme (barriers to participation have previously been reported ${ }^{15}$ ), or management of cases by non-consultant ophthalmologists (who do not receive BOSU reporting cards). To adequately assess completeness of reporting, an alternative routine source to identify all cases would be necessary, but none was available. The mean BOSU card return rate for the 12-month study was $79 \%$. This shows a high level of participation in the BOSU reporting scheme and was used to estimate the corrected annual incidence of IOFB in the UK. 

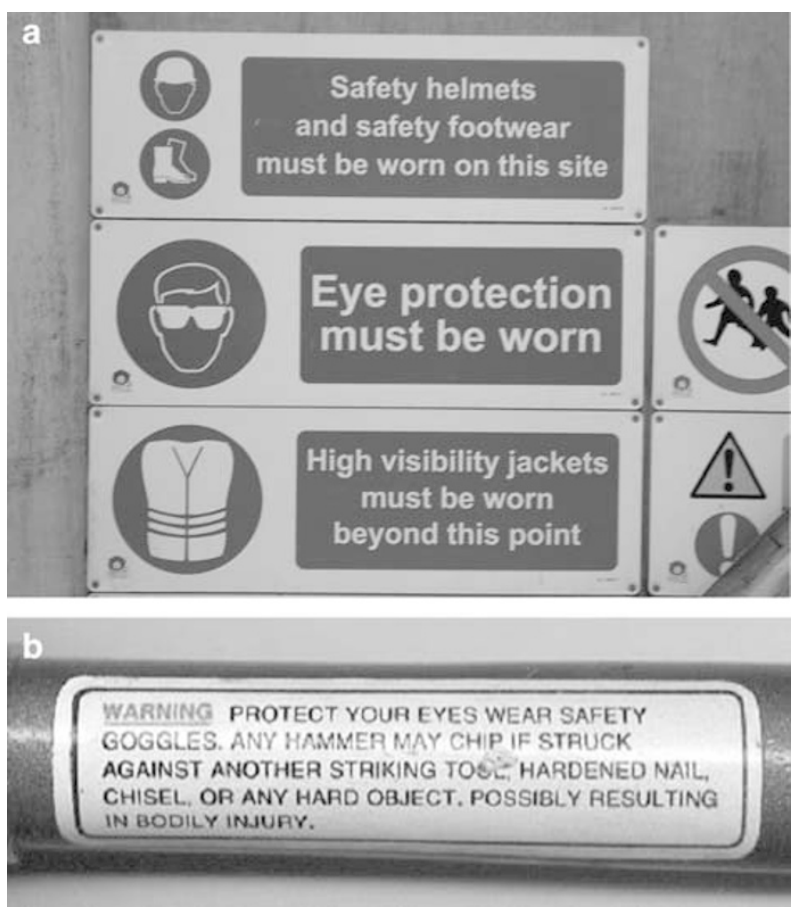

Figure 1 (a) Safety sign at the construction site. (b) Warning label on the shaft of hammer.

Factors contributing to a reduction in the number of IOFBs in the UK could be reduced exposure to risk through a shift away from manufacturing towards service industry jobs and increased use of protective eyewear at work and home. Many employees are reminded to wear eye protection through warning notices at places of work (see Figure 1a). The majority of hammers sold in the United Kingdom now have either a written warning label (see Figure 1b) or a symbol of a person wearing safety goggles; however, in this study, only $5 \%$ of patients reported wearing protective eyewear at the time of injury. This is similar to the $6 \%$ reportedly wearing eye protection in a large American study of IOFBs. ${ }^{9}$ Lack of eye protection is obviously a significant risk for IOFB, but it is also important to ensure that protective eyewear adequately shields the eyes from all angles of trajectory of foreign bodies (ie goggles or spectacles with side guards).

Previous studies have reported that $50-54 \%$ of IOFBs are work-related injuries, ${ }^{3,6,9}$ and hammering is the most common mechanism of injury (71-80\% of cases). ${ }^{7,10-12}$ In this study, $62 \%$ of injuries occurred during paid employment and the mechanism of injury was hammering in $62 \%$ and machine tools in $19 \%$. These results demonstrate that the majority of IOFBs could be prevented if employers and employees followed the instructions of the UK Health and Safety at Work Act (1974) to provide, and appropriately use, protective eyewear when hammering or using machine tools. For the $13 \%$ of injuries that occurred at home during DIY (do it yourself), it is the responsibility of the individual to protect themselves.

In this study, the IOFB was found in the anterior segment in $24 \%$, the posterior segment in $73 \%$, and involved both segments in 3\%. This is a similar anatomical distribution to previous studies where posterior segment IOFBs occurred in $59-88 \%$ of cases. ${ }^{3,6-8,11,12}$ Anterior segment IOFBs are often directly visible and can be removed with forceps or aspiration. Posterior segment IOFBs can be removed by an external (scleral incision and use of an electromagnet over the incision) or internal approach (pars plana vitrectomy and use of internal magnet or forceps). In rare cases, a combination of techniques is used at the same procedure. In this study, pars plana vitrectomy was the most frequently used technique for removal of posterior segment IOFB $(88 \%$ $(53 / 60))$. The external magnet was utilised in 17\% (10/60) of posterior segment cases (in half of these cases $(5 / 60)$ in combination with pars plana vitrectomy).

The external approach has been advocated by some as an initial strategy for all cases except those with known vitreoretinal pathology (such as retinal detachment). ${ }^{11}$

Other authors have suggested that the external approach be restricted to cases with small $(<3 \mathrm{~mm})$ foreign bodies located within the vitreous or minimally embedded in the retina. ${ }^{13}$ Pars plana vitrectomy has been recommended in the case of foreign bodies that are poorly visualised (owing to cataract or vitreous haemorrhage) and that are encapsulated in the retina, and in patients with concurrent intraocular damage (such as retinal detachment) or in whom endophthalmitis is suspected. ${ }^{10} \mathrm{~A}$ retrospective study of external vs internal approach found no statistically significant difference in visual outcome and suggested that the external approach remained a reasonable option in selected cases. ${ }^{19}$ This study also found no significant difference between these two approaches, but the number of posterior segment IOFBs removed with the external magnet was small $(10 / 60)$.

During the study period, UK ophthalmologists favoured pars plana vitrectomy over an external approach for removal of posterior segment IOFBs. As the majority of IOFBs $(74 \%)$ were removed by specialist vitreoretinal surgeons, it would appear that general ophthalmologists are not attempting to remove posterior segment IOFBs with the external electromagnet. This may mean that the external electromagnet is a redundant piece of equipment in many ophthalmology departments and may only be required in centres that offer specialist vitreoretinal surgery.

Endophthalmitis was diagnosed in 9\% of cases in this study - in all cases, endophthalmitis was suspected before IOFB removal. Previous studies have reported 
rates of endophthalmitis following IOFB of $0-13 \% .^{8,9,20,21}$ In a study of 492 cases of IOFB in the United States, endophthalmitis was diagnosed in $6.9 \%$, of which $91.2 \%$ had signs of infection at the time of removal of the IOFB. ${ }^{9}$ There is consensus of opinion that intravitreal antiobiotics should be administered when there is clinical suspicion of endophthalmitis; ${ }^{8,22}$ however, the benefit of routine prophylactic intravitreal antibiotics in all cases of IOFB has not been demonstrated. Studies of penetrating injuries (including those with IOFB) have failed to show a statistically significant benefit of prophylactic intraocular antibiotics. ${ }^{21,23}$ In a recent study of 55 cases of IOFB sustained by US military personnel, no patients received intravitreal antibiotics and there were no cases of endophthalmitis. ${ }^{20}$ The use of prophylactic intravitreal antibiotics when there is a delay of $24 \mathrm{~h}$ or more in removing the IOFB has been proposed. Such a delay was associated with an increased risk of endophthalmitis in one study, ${ }^{9}$ but this was not found to be a significant factor in subsequent work. ${ }^{21}$

Best-corrected visual acuity of the injured eye at final follow-up was $6 / 12$ or better in $67 \%, 6 / 18$ to $6 / 60$ in $11 \%$, and worse than $6 / 60$ in $22 \%$. This distribution of visual outcome is very similar to previous studies of anterior and posterior segment IOFB, with the majority (61-71\%) of patients maintaining good visual acuity $(6 / 12$ or better) but a significant minority (17-38\%) sustaining severe loss of vision (worse than $6 / 60) .5,8,11$ If bestcorrected vision has been measured with a pinhole, or with a correcting lens that the patient does not routinely wear, day-to-day functional vision is often poorer than best-corrected vision. This is particularly true for aphakic eyes and those filled with silicone oil. In this study, when day-to-day vision was used to assess visual outcome, the number of eyes with vision worse than 6/60 increased from 22 to $34 \%$. Loss of accommodation is another factor that is often forgotten when assessing visual outcome. In this study, the crystalline lens had been removed in 56\% $(48 / 85)$ of patients at follow-up, the majority of whom would have had useful accommodation before their injury (74\% of patients were less than 50 years old).

In this study, the prognostic factors for a poor visual outcome included poor visual acuity at presentation, development of endophthalmitis, development of retinal detachment, prolapse of intraocular tissue, and large size of IOFB. Many of these factors have previously been reported, ${ }^{6-8,10,13,14}$ and indicate that severe initial damage is strongly predictive of poor visual outcome. Interestingly, removal of IOFB within $24 \mathrm{~h}$ of presentation, RAPD in injured eye at presentation, and IOFB involving the posterior segment were not found to be significant factors for a poor visual outcome.

Despite poor vision at presentation being predictive of a poor outcome, many patients with poor presenting vision had a good outcome. The number of patients with vision worse than $6 / 60$ was $45 \%$ at presentation and $22 \%$ at follow-up. Current management of IOFB in the United Kingdom can lead to a good outcome in many patients but there remain a significant number of patients who, despite best management, sustain long-term severe visual loss. Prevention is the most important way to reduce the burden of visual loss arising from IOFB. The reduced incidence of IOFB in this study, compared to previous studies, suggests that warnings to wear protective eyewear might be more conspicuous than in the past and, as a result, more people are adhering to this advice. Individuals often ignore health and safety advice if the perceived risk of injury is low and there is an element of inconvenience in the method of risk reduction. (How many doctors have taken a blood sample without wearing gloves?) The fact that only $5 \%$ of patients were wearing protective eyewear at the time of injury in this study emphasises the need to conspicuously display warnings of eye injury arising from the use of hammers and machine tools. It is then the responsibility of the individual to follow or ignore the advice.

\section{Acknowledgements}

We are very grateful for the support of the British Ophthalmological Surveillance Unit. This work was supported by a grant from the Speed Pollock Memorial Trust, for which we are grateful. We thank the following ophthalmologists who reported cases in this study: Miss G Adams, Mr S Ahmed, Mr S Amjad, Mr H Bacon, Mr Baranyovits, Dr C Barras, Mr A Bates, Mr R Bates, Mr M Batterbury, Mr G Bedford, Miss K Belfer, Mr L Benjamin, Mr R Best, Mr M Birch, Mr J Butcher, Mr A Callear, Mr C Canning, Miss P Chakraborthy, Mr S Charles, Mr D Charteris, Mr H Chen, Mr M Clarke, Mr A Coombes, Mr D Cottrell, Mr E Davies, Miss D Depla, Mr J Diamond, Prof A Dick, Mr R Downes, Mr A Elliott, Miss B Enoch, Mr E Ezra, Mr I Fawcett, Mr A Fayi-Waboso, Mr A W Fitt, Mr D Flanagan, Miss J Forbes, Dr A Gaskell, Mr F Ghanchi, Mr J Gillow, Mr R Goble, Mr C Gorman, Mr R Gray, Mr M Greaney, Mr A Greenwood, Col M Griffiths, Mr C Groenwald, Mr E G Hale, Dr H Hammer, Mrs B Harney, Miss R Harrison, Mr P Hassett, Mr R Haynes, Mr M Headon, Mr C Heaven, Mr M Hero, Mr R Hill, Mr Hiranandani, Mr C Hutchison, Mr D Inglesby, Mr M Jabir, Mr D Jones, Mr V Kayarkar, Dr P Kearns, Mr S Kelly, Mr G Kirkby, Mr J Kirkpatrick, Mr R Ling, Miss N Lois, Mr T Manners, Mr R Markham, Mr P McCormack, Mr C McLean, Mr R McPherson, Mr T Metcalfe, Miss B Mulholland, Mr D Mulholland, Dr J Murdoch, Dr R Murray, Mr C Neoh, Mr M Neugebauer, Mr D Newman, 
Mr A O’Driscoll, Mr C Patel, Ms S Porter, Dr B Power, Mr N Price, Dr A Pyott, Miss T Richardson, Mr M Rifaat, Mr J Sharkey, Mr R Sheard, Ms G Silvestri, Dr J Singh, Mr A Smith, Mr R Smith, Mr J Stevens, Mr C Stevenson, Mr P Sullivan, Mr V Tanner, Mr K Toor, Mr S Tuft, Mr A Tyers, Mr S Vardy, Mr G Walters, Mrs S Webber, Mr J West, Mr S White, Dr I Whyte, Mr T Williamson, Mr S Winder, and Mr W Woon.

\section{References}

1 Wong TY, Tielsch JM. A population-based study on the incidence of severe ocular trauma in Singapore. Am J Ophthalmol 1999; 128: 345-351.

2 Desai P, MacEwen CJ, Baines P, Minassian DC. Epidemiology and implications of ocular trauma admitted to hospital in Scotland. J Epidemiol Commun Health 1996; 50: 436-441.

3 Punnonen E, Laattikainen L. Prognosis of perforating eye injuries with intraocular foreign bodies. Acta Ophthalmol 1989; 66: 483-491.

4 Pieramici DJ, MacCumber MW, Humayun MU, Marsh MJ, de Juan Jr E. Open-globe injury. Update on types of injuries and visual results. Ophthalmology 1996; 103: 1798-1803.

5 Percival SPB. A decade of intraocular foreign bodies. Br J Ophthalmol 1972; 56: 454-461.

6 Chiquet C, Zech J-C, Denis P, Adeleine P, Trepsat C. Intraocular foreign bodies. Factors influencing final visual outcome. Acta Ophthalmol Scand 1999; 77: 321-325.

7 Armstrong MFJ. A review of intraocular foreign body injuries and complications in N. Ireland from 1978-1986. Int Ophthalmol 1988; 12: 113-117.

8 Greven CM, Engelbert NE, Slusher SS, Nagy SS. Intraocular foreign bodies. Management, prognostic factors and visual outcomes. Ophthalmology 2000; 107: 608-612.

9 Thompson JT, Parver LM, Enger CL, Mieler WF, Liggett PE. Infectious endophthalmitis after penetrating injuries with retained intraocular foreign bodies. Ophthalmology 1993; 100: 1468-1474.

10 Abu El-Asrar AM, Al-Amro SA, Khan NM, Kangave D. Visual outcome and prognostic factors after vitrectomy for posterior segment foreign bodies. Eur J Ophthalmol 2000; 10: 304-311.
11 Hadden OS, Wilson JL. The management of intraocular foreign bodies. Aust NZ J Ophthalmol 1990; 18: 343-351.

12 Behrens-Baumann W, Praetorius G. Intraocular foreign bodies. 297 consecutive cases. Ophthalmologica 1989; 198: 84-88.

13 Chiquet C, Zech JC, Gain P, Adeleine P, Trepsat C. Visual outcome and prognostic factors after magnetic extraction of posterior segment foreign bodies in 40 cases. Br J Ophthalmol 1998; 82: 801-806.

14 Wani VB, Al-Ajmi M, Thalib L, Azad RV, Abul M, Al-Ghanim $\mathrm{M}$ et al. Vitrectomy for posterior segment intraocular foreign bodies: visual results and prognostic factors. Retina 2003; 23: 654-660.

15 Foot B, Stanford M, Rahi J, Thompson J. The British Ophthalmological Surveillance unit: an evaluation of the first 3 years. Eye 2003; 17: 9-15.

16 Thacker SB, Redmond S, Rothenberg RB, Spitz SB, Choi K, White MC. A controlled trial of disease surveillance strategies. Am J Prev Med 1986; 2: 345-350.

17 Vogt RL, LaRue D, Klaucke DN, Jillison DA. Comparison of an active and passive surveillance system of primary care providers for hepetitis, measles, rubella, and salmonellosis in Vermont. Am J Pub Health 1983; 73: 795-797.

18 Asch DA, Jedrziewski MK, Christakis NA. Response rates to mail surveys published in medical journals. J Clin Epidemiol 1997; 50: 1129-1136.

19 Chow DR, Garretson BR, Kuczynski B, Williams GA, Margherio R, Cox MS et al. External versus internal approach to the removal of metallic intraocular foreign bodies. Retina 2000; 20: 364-369.

20 Thach AB, Ward TP, Dick II JSB, Bauman WC, Madigan Jr WP, Goff MJ et al. Intraocular foreign bodies during Operation Iraqi Freedom. Ophthalmology 2005; 112: 1829-1833.

21 Essex RW, Yi Q, Charles PGP, Allen PJ. Post-traumatic endophthalmitis. Ophthalmology 2004; 111: 2015-2022.

22 Reynolds DS, Flynn Jr HW. Endophthalmitis after penetrating ocular trauma. Curr Opin Ophthalmol 1997; 8: 32-38.

23 Soheilian M, Nafati N, Peyman GA. Prophylaxis of acute posttraumatic bacterial endophthalmitis with or without combined intraocular antibiotics: a prospective double-masked randomised pilot study. Int Ophthalmol 2001; 24: 323-330. 